\title{
Shortcuts to Adiabaticity for the Quantum Rabi Model: Efficient Generation of Giant Entangled cat States via Parametric Amplification
}

\author{
Ye-Hong Chen, ${ }^{1}$ Wei Qin, ${ }^{1}$ Xin Wang, ${ }^{1,2}$ Adam Miranowicz, ${ }^{1,3}$ and Franco Nori ${ }^{1,4}$ \\ ${ }^{1}$ Theoretical Quantum Physics Laboratory, RIKEN Cluster for Pioneering Research, Wako-shi, Saitama 351-0198, Japan \\ ${ }^{2}$ Institute of Quantum Optics and Quantum Information, \\ School of Science, Xi'an Jiaotong University, Xi'an 710049, China \\ ${ }^{3}$ Faculty of Physics, Adam Mickiewicz University, 61-614 Poznań, Poland \\ ${ }^{4}$ Department of Physics, University of Michigan, Ann Arbor, Michigan 48109-1040, USA
}

(Dated: January 15, 2021)

\begin{abstract}
We propose a method for the fast generation of nonclassical ground states of the Rabi model in the ultrastrong and deep-strong coupling regimes via the shortcuts-to-adiabatic (STA) dynamics. The time-dependent quantum Rabi model is simulated by applying parametric amplification to the Jaynes-Cummings model. Using experimentally feasible parametric drive, this STA protocol can generate large-size Schrödinger cat states, through a process that is $\sim 10$ times faster compared to adiabatic protocols. Such fast evolution increases the robustness of our protocol against dissipation. Our method enables one to freely design the parametric drive, so that the target state can be generated in the lab frame. A largely detuned light-matter coupling makes the protocol robust against imperfections of the operation times in experiments.
\end{abstract}

Keywords: Quantum Rabi model; Shortcuts to adiabaticity; Nonclassical state

Introduction.-The quantum Rabi model [1-3] is the lowest-dimensional Hamiltonian describing the lightmatter interaction beyond the rotating-wave approximation (RWA),

$$
H_{\mathrm{R}}=\omega_{c} a^{\dagger} a+\frac{\omega_{q}}{2} \sigma_{z}+\sigma_{x}\left(g a^{\dagger}+g^{*} a\right) . \quad(\hbar=1)
$$

Here, $\omega_{c}\left(\omega_{q}\right)$ is the frequency of the cavity (qubit), $g$ is the light-matter coupling strength, $a^{\dagger}(a)$ is the creation (annihilation) operator of the cavity field, and $\sigma_{x}$ and $\sigma_{z}$ are Pauli operators of the qubit. This model was first introduced 90 years ago and it has been used to describe the dynamics of a wide variety of physical setups [4], ranging from quantum optics to condensed matter physics. The popular models of Dicke [5], Hopfield [6], and Tavis-Cummings [7] are just multiqubit generalizations of the Rabi model, while the JaynesCummings (JC) model [8] is its simplified version [9]. Generally, the Rabi model can be divided into different coupling regimes [9-11], according to the normalized coupling strength $\eta=g / \omega_{c}$. When focusing on the ultrastrong $(|\eta| \simeq 0.1 \sim 1)$ and deep-strong $(|\eta| \gtrsim 1)$ regimes, the counterrotating terms in $H_{\mathrm{R}}$ cannot be neglected. This leads to areas of unexplored physics and gives rise to many fascinating quantum phenomena, such as the asymmetry of vacuum Rabi splitting [12], nonclassical photon statistics [13, 14], and superradiance transition [15-18].

For instance, the ground state of the Rabi model is a squeezed-vacuum state and involves virtual cavity photons $[14,19,20]$. Specifically, when $\omega_{q} \ll g$, the ground state of the Rabi model is

$$
|G\rangle=\frac{1}{2}\left(\mathcal{N}_{+}|g\rangle\left|\mathrm{cat}_{+}\right\rangle-\mathcal{N}_{-}|e\rangle\left|\mathrm{cat}_{-}\right\rangle\right)
$$

which is an entangled Schrödinger cat state. Here, $\mathcal{N}_{ \pm}=\sqrt{2\left[1 \pm \exp \left(-2|\eta|^{2}\right)\right]}$ determine the probability amplitudes of the even $(+)$ and odd $(-)$ cat states $\mid$ cat $\left._{ \pm}\right\rangle=(|\eta\rangle \pm|-\eta\rangle) / \mathcal{N}_{ \pm}$, respectively. The states $| \pm \eta\rangle$ are coherent states. The state $|g\rangle(|e\rangle)$ is the ground (excited) state of the qubit. By imposing the system to be in this ground state, one can generate the maximally entangled cat state $(\mathrm{MECS})$ when $\mathcal{N}_{+} \simeq \mathcal{N}_{-}$. The generation of the MECSs is significant not only for the demonstration of the fundamentals of quantum physics, but also has wide applications in modern quantum technologies, such as quantum information processing [21-25] and quantum metrology [26]. For instance, giant cat qubits are very robust against photon dephasing, so that they can be very promising for fault-tolerant quantum computation [22-24].

To generate the MECS, the system needs to enter the deep-strong coupling (DSC) regime of $|\eta| \gtrsim \sqrt{2}$, which is, however, still difficult to achieve in experiments [27-36]. Researchers are encouraged to use simulation protocols [37-48] based on the JC model [49-51] to study exotic phenomena in the DSC regime. For instance, using linear [37] or nonlinear drives [41, 42], one can modify the sideband of a cavity-qubit coupled system, so as to enhance the effective light-matter coupling to enter the DSC regime. This opens the possibility to adiabatically control the effective coupling strength based on, e.g., a time-dependent parametric drive, to prepare the target state $|G\rangle$ in the squeezed-light frame [42]. However, the adiabatic control requires a very small changing rate in the control parameters, usually leading to a long-time evolution. Such a long-time evolution inevitably increases the effect of dissipation, resulting in a low-fidelity target state. In addition, how to turn off the parametric drive without affecting the prepared 
entangled state is still an open problem.

In this Letter, we propose to use shortcuts-to-adiabatic (STA) methods [52-63], e.g., counterdiabatic (CD) driving, to rapidly generate the target state $|G\rangle$. The STA methods are a series of protocols mimicking adiabatic dynamics beyond the adiabatic limit and have been widely applied for quantum state engineering [64-79]. Specifically, the CD driving [55, 56] enables controlling a quantum system, such that the system can accurately evolve along an adiabatic path (e.g., an instantaneous eigenstate of the reference Hamiltonian) beyond the adiabatic limit, where nonadiabatic excitations can be precisely compensated by, e.g., adding an auxiliary driving term to a reference Hamiltonian [80]. Using the STA method allows us to significantly shorten the evolution time as compared to the adiabatic protocol. Thus, we can suppress the effect of dissipation and significantly improve the fidelity of a given state. The parametric drive can be smoothly turned off in our STA protocol, because the amplitudes of the parametric drive are continuously turnable. Additionally, the discussed model is generic, so our proposal can be realized in many physical systems, in particular, circuit quantum electrodynamics (QED) systems [41, 42, 78] or ion traps $[45,47]$.

Adiabatic limit.-Assuming $\omega_{q} \ll g$ and $H_{\mathrm{R}} \equiv H_{\mathrm{R}}(t)$ [with a controllable parameter $\eta \equiv \eta(t)$ ] to be time dependent, the Rabi Hamiltonian in Eq. (1) can be diagonalized by the unitary operator $[9,10]$

$$
U(t)=\left|+{ }_{x}\right\rangle\left\langle+{ }_{x}|D[-\eta(t)]+|{ }_{x}\right\rangle\left\langle-{ }_{x}\right| D[\eta(t)],
$$

where $\left| \pm_{x}\right\rangle$ are the eigenstates of $\sigma_{x}$ and $D[\eta(t)]=$ $\exp \left[\eta(t) a^{\dagger}-\eta^{*}(t) a\right]$ is the displacement operator. To avoid the nonadiabatic transitions between the instantaneous eigenstates $\left\{\left|E_{m}(t)\right\rangle\right\}$ [eigenvalues $\xi_{m}(t)$ ] of $H_{\mathrm{R}}(t)$, the system needs to satisfy the adiabatic condition $\left|\left\langle E_{m}(t) \mid \dot{E}_{n \neq m}(t)\right\rangle\right| \ll\left|\xi_{m}(t)-\xi_{n}(t)\right|$.

$C D$-driving Hamiltonian.-According to Eq. (3) and Berry's transitionless algorithm [56], the CD-driving Hamiltonian for the reference Hamiltonian $H_{\mathrm{R}}(t)$ is

$$
H_{\mathrm{CD}}(t)=i \dot{U}(t) U^{\dagger}(t)=i \sigma_{x}\left[\dot{\eta}^{*}(t) a-\dot{\eta}(t) a^{\dagger}\right] .
$$

The desired STA process can be realized by adding the CD-driving Hamiltonian $H_{\mathrm{CD}}(t)$ into the reference Hamiltonian $H_{\mathrm{R}}(t)$ to construct a feasible total Hamiltonian: i.e., $H_{\text {tot }}(t)=H_{\mathrm{R}}(t)+H_{\mathrm{CD}}(t)$ [57]. In this case, we can predict an ideal evolution along the instantaneous eigenstate $\left|E_{m}(t)\right\rangle$, as $H_{\text {tot }}(t)$ ideally satisfies the Schrödinger equation $i\left|\dot{E}_{m}(t)\right\rangle=\left[\xi_{m}(t)+\right.$ $\left.H_{\mathrm{CD}}(t)\right]\left|E_{m}(t)\right\rangle[68]$. Thus, assuming the initial state to be $\left|E_{0}(0)\right\rangle=|g\rangle|0\rangle$, we obtain the target state $\left|E_{0}\left(t_{f}\right)\right\rangle=$ $|G\rangle$ at the final time $t_{f}$. However, realizing a timedependent Rabi model in the DSC regime is still a major challenge in experiments. In the following, we illustrate how to simulate $H_{\text {tot }}(t)$ based on a parametrically driven

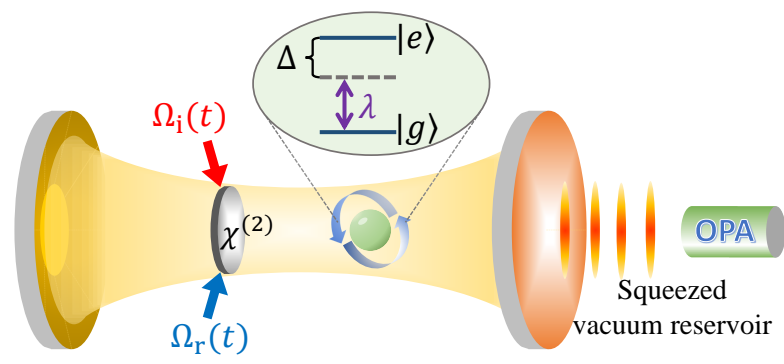

FIG. 1. Schematic illustration of a cavity QED system containing a single-mode cavity, a qubit, a $\chi^{(2)}$-nonlinear medium, and an optical parametric amplifier (OPA). The qubit $(|g\rangle=$ ground state and $|e\rangle=$ excited state) coupled to the cavity with coupling strength $\lambda$ and large detuning $\Delta$. The nonlinear drive $\Omega_{\mathrm{r}}(t)$ induces a time-dependent squeezed-cavity mode. The other nonlinear drive $\Omega_{\mathrm{i}}(t)$ $\left[\pi / 2\right.$-dephased from $\left.\Omega_{\mathrm{r}}(t)\right]$ is applied to counteract the nonadiabatic transition induced by mapping the system dynamics into the time-dependent squeezed-light frame. The OPA is used to generate a squeezed-vacuum reservoir, which couples to the cavity mode to minimize the influence of the squeezing-induced noise.

JC model, so as to realize the STA protocol and generate the state $|G\rangle$.

Model and effective Hamiltonian.-As shown in Fig. 1, our STA proposal is realized in the JC model. The cavity is subjected to two time-dependent (two-photon) drives, with the same frequency $\omega_{p}$, but with different real amplitudes, $\Omega_{\mathrm{r}}(t)$ and $\Omega_{\mathrm{i}}(t)$. The drive $\Omega_{\mathrm{i}}(t)$ is $\pi / 2$-dephased from $\Omega_{\mathrm{r}}(t)$. The Hamiltonian in a frame rotating at $\omega_{p} / 2$ reads

$$
H_{0}(t)=\Delta a^{\dagger} a-\left[\frac{\Omega_{\mathrm{r}}(t)+i \Omega_{\mathrm{i}}(t)}{2} a^{2}-\lambda a^{\dagger} \sigma+\text { h.c. }\right],
$$

where $\Delta=\omega_{c}-\omega_{p} / 2, \sigma=|g\rangle\langle e|, \lambda \ll \omega_{\mathrm{c} / \mathrm{q}}$ is the qubitcavity coupling strength, and we have assumed $\omega_{q}=$ $\omega_{p}$. By performing the unitary transformation $S(t)=$ $\exp \left[r(t)\left(a^{\dagger 2}-a^{2}\right) / 2\right]$, with $r(t)$ satisfying tanh $[2 r(t)]=$ $\Omega_{\mathrm{r}}(t) / \Delta$, we obtain the effective Hamiltonian

$$
H_{S}(t) \approx \Delta \operatorname{sech}[2 r(t)] a^{\dagger} a+\lambda e^{r(t)} \sigma_{x}\left(a^{\dagger}+a\right) / 2,
$$

where we have neglected the undesired terms by assuming $\Omega_{\mathrm{i}}(t)=\dot{r}(t)$ and $\lambda \ll \Delta$. The condition $\Omega_{\mathrm{i}}(t)=\dot{r}(t)$ has been applied according to the transitionless algorithm to counteract the nonadiabatic transition caused by the time-dependent unitary transformation $S(t)$ (see the Supplemental Material [81] for details). The effective normalized coupling strength of $H_{S}(t)$ is

$$
\tilde{\eta}(t)=\frac{\lambda}{4 \Delta}\{\exp [3 r(t)]+\exp [-r(t)]\}
$$

To show the advantages of our STA protocol, as compared to the adiabatic scheme [42], in the following discussion we denote $\tilde{*}$ and $*(*=\eta, \lambda, r, \ldots)$ to represent 

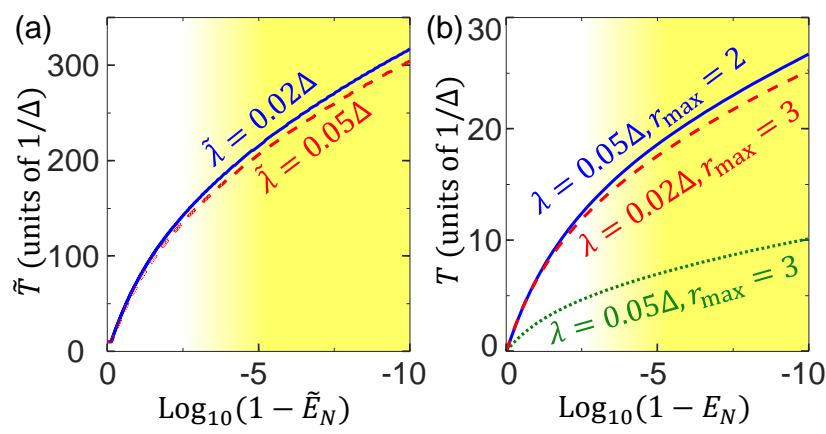

FIG. 2. Total evolution time required for (a) the adiabatic process and (b) the STA process, to achieve the MECS versus the desired entanglement cost [characterized by the logarithmic negativity $\left.\tilde{E}_{N}\left(E_{N}\right)\right]$. For (a), the squeezing parameter is $\tilde{r}(t)=\tilde{r}_{\max } /\{1+\exp [\tilde{f}(t)]\}$, where $\tilde{r}_{\max }$ is the peak value of $\tilde{r}(t)$ and $\tilde{f}(t)=\tilde{f}_{0}(1 / 2-t / \tilde{T})$, with $\tilde{f}_{0}=10$. For (b), the parameters are $\eta(0)=(1+i) / 100$ and $r(t)=r_{\max } /\{1+\exp [f(t)]\}$, with $f(t)=f_{0} \cos (2 \pi t / T)$ and $f_{0}=10$, resulting in $r(0)=r\left(t_{f}\right) \simeq 0$ and $\dot{r}(0)=\dot{r}\left(t_{f}\right) \simeq 0$. The light-matter coupling $(\tilde{\lambda}, \lambda \ll \Delta)$ are chosen to satisfy the condition to neglect the undesired terms to obtain the effective Hamiltonian in Eq. (6). The comparison between the panels shows that, the time required in the STA process to achieve the target state is $\sim 10$ times shorter than that required in the adiabatic process. The yellow-shaded area in each panel shows $\left(\tilde{E}_{N}, E_{N} \gtrsim 99 \%\right)$, indicating that the target state in this area is maximally entangled.

all the parameters in the adiabatic and STA processes, respectively. Here, $\tilde{*}$ and $*$ have the same physical meaning.

Adiabatic protocol.-When $|\dot{\tilde{\eta}}(t)| \ll \Delta \operatorname{sech}[2 \tilde{r}(t)]$, one can achieve the adiabatic evolution along the ground eigenstate of $H_{S}(t)$ [42]. The adiabatic condition requires $\dot{\tilde{r}}(t) / \Delta \rightarrow 0$, thus leading to slow evolution. Figure 2(a) shows the relationship between the total evolution time $\tilde{T}$ and the logarithmic negativity $\tilde{E}_{N}=\log _{2}\left\|\rho^{\Gamma_{\mathrm{q}}}\right\|_{1}[82]$ of the adiabatic process. Here, $\Gamma_{\mathrm{q}}$ denotes the partial transpose with respect to the qubit, and $\|\cdot\|_{1}$ the trace norm. The evolution time $\tilde{T}$ significantly increases when the desired entanglement cost grows. To achieve the MECS with $\tilde{E}_{N} \gtrsim 99.99 \%$, one needs $\tilde{T} \gtrsim 200 / \Delta$ via the adiabatic process.

According to Eq. (7), a fixed final squeezing parameter $\tilde{r}\left(t_{f}\right)=\tilde{r}_{\max }$ is needed to obtain the target state $|G\rangle$. As a result, the MECS only can be prepared in the squeezed-light frame rather than the lab frame, i.e., the final state is $S\left(t_{f}\right)|G\rangle$. To obtain a MECS in the lab frame, one needs to turn off the parametric drive immediately when $t>t_{f}$. However, rapidly decreasing the squeezing parameter $r(t)$ induces an undesired nonadiabatic transition, which pumps many photons into the cavity in a very short time [81]. Then, the final state might be unpredictable.

$S T A$ protocol.-We assume $H_{\text {tot }}(t)=H_{S}(t)$, resulting
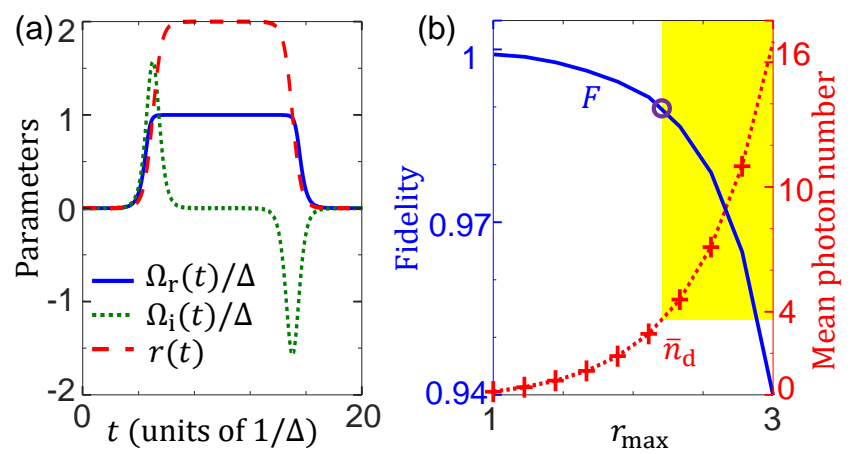

FIG. 3. STA protocol when $\eta(0)=(1+i) / 100, \lambda=0.045 \Delta$, and $T=20 / \Delta$ : (a) Finite-duration drives $\Omega_{\mathrm{r}}(t), \Omega_{\mathrm{i}}(t)$ and squeezing parameter $r(t)$ when the peak squeezing parameter $r_{\max }=2$. (b) Fidelity (the blue vertical axis on the left side) of the target state versus $r_{\max }$. The red vertical axis on the right side denotes the mean photon number $\bar{n}_{\mathrm{d}}$ (red-dotted curve with marks "+") of the target state. The yellow-shaded area in (b) shows $\bar{n}_{\mathrm{d}} \geq 4$, indicating that the target state in this area can be called a large-size entangled cat state. To generate the large-size entangled cat state with $F \gtrsim 99 \%$ and $\bar{n}_{\mathrm{d}} \simeq 4.3$, one can choose $r_{\max }=2.3$ (the purple circle).

in $\Delta \operatorname{sech}[2 r(t)]: \Rightarrow \omega_{c}$ and $\lambda \exp [r(t)]: \Rightarrow 2[g-i \dot{\eta}(t)]$, where $\eta(t)=g / \omega_{c}$. Thus, we obtain the equations of motion for the coherent state amplitude $\eta(t)$ :

$$
\begin{aligned}
& \operatorname{Re}[\dot{\eta}(t)]=\Delta \operatorname{Im}[\eta(t)] \operatorname{sech} 2 r(t), \\
& \operatorname{Im}[\dot{\eta}(t)]=\frac{\lambda}{2} \exp [r(t)]-\Delta \operatorname{Re}[\eta(t)] \operatorname{sech} 2 r(t),
\end{aligned}
$$

where $\operatorname{Re}[*](\operatorname{Im}[*])$ denotes the real (imaginary) part of the parameter " $*$." Note that $\eta(t)=g / \omega_{c}$ is different from the definition of $\tilde{\eta}(t)$ in Eq. (7), thus the Hamiltonian $H_{S}(t)$ can drive the system to evolve along the ground eigenstate $\left|E_{0}(t)\right\rangle$ of the Hamiltonian $H_{\mathrm{R}}(t)$. According to Eq. (8), $\eta(t)$ relies on the time integration of the squeezing parameter $r(t)$. This allows to rapidly achieve a large value of $\eta\left(t_{f}\right)$ without any restrictions on the final squeezing parameter $r\left(t_{f}\right)$. Thus, the STA process can achieve the target state $|G\rangle$ in the lab frame, i.e., $r\left(t_{f}\right)=0$.

In Fig. 2(b), we display the total evolution time $T$ required for the STA process to obtain the target state versus the logarithmic negativity $E_{N}$. We find that $T$ is significantly shortened when we increase the coupling strength $\lambda$ and the peak squeezing parameter $r_{\max }$. For an experimentally feasible gain of $10 \log _{10}\left[\exp \left(2 r_{\max }\right)\right] \sim$ $20 \mathrm{~dB}$ [83-85] (corresponding to $r_{\max } \sim 2.3$ ), the evolution time to achieve the MECS with $E_{N} \gtrsim 99.99 \%$ via the STA process is $T \sim 20 / \Delta$, which is $\sim 10$ times shorter than that via the adiabatic process.

In the above numerical calculation of Fig. 2(b), we have used the parameter $r(t)=r_{\max } /\{1+\exp [f(t)]\}$ with $f(t)=f_{0} \cos (2 \pi t / T)$, where $f_{0} \gg 1$ controls the initial and final values of the squeezing parameter $r(t)$. 
With these parameters, the pulses $\Omega_{\mathrm{r}}(t)$ and $\Omega_{\mathrm{i}}(t)$ have finite durations, so that we can smoothly turn off the parametric drive [see Fig. 3(a)].

In Fig. 3(b), we show the desired mean photon number $\bar{n}_{\mathrm{d}}=\left\langle G\left|a^{\dagger} a\right| G\right\rangle$ versus the peak squeezing parameter $r_{\max }$ (red-dotted curve with "+"). We find that, for a fixed evolution time $T, \bar{n}_{\mathrm{d}}$ increases sharply when $r_{\max }$ increases. Experimentally, a parametric gain of $\sim 20 \mathrm{~dB}$ $\left(r_{\max } \sim 2.3\right)$ has been achieved [45], and $\sim 30 \mathrm{~dB}$ has also been predicted under experimentally feasible conditions [83-85]. These realistic parameters allow for generating a high-fidelity $(F>90 \%)$ target state with $\bar{n}_{\mathrm{d}}=4 \sim 10$ (large-amplitude nonclassical states), as shown by the blue, solid curve in Fig. 3(b). Here, the fidelity of the state $|G\rangle$ is defined as $F=\left|\left\langle G\left|\rho\left(t_{f}\right)\right| G\right\rangle\right|$. When $r_{\max }=2.3$ and $\lambda=0.045 \Delta$, we find that the target state $|G\rangle$ can be generated with $F \simeq 99 \%$ and $\bar{n}_{\mathrm{d}} \simeq 4$ [see purple circle in Fig. 3(b)].

Robustness of the STA approach - In the following, we focus on discussing the robustness of the STA protocol when $r_{\max }=2.3$ and $\lambda=0.045 \Delta$. We first assume the imperfection of a parameter $*$ as $\delta *=*^{\prime}-*$, where $*^{\prime}$ and $*$ denote the actual and ideal values, respectively. Because of large detuning $\lambda \ll \Delta$, when the parametric drive vanishes, the mean photon number and the entanglement of the system can remain unchanged for a long time in the absence of dissipation. Thus, our STA protocol is robust against the imperfect parameters of the total evolution time. As shown in Fig. 4(a), a $20 \%$ imperfection of the total evolution time only causes $\lesssim 1 \%$ and $\lesssim 5 \%$ changes of the logarithmic negativity $E_{N}$ and the mean photon number $\bar{n}_{\mathrm{d}}$, respectively.

Then, we compare the entanglement preparation via the STA and the adiabatic processes in the presence of cavity and qubit losses. Because of the relatively strong squeezing, the difference of the frequencies of the photons $\left(\omega_{p} / 2\right)$ pumped by the two-photon drives and those of the squeezed-cavity mode $\left(\omega_{p} / 2+\Delta \operatorname{sech}[2(r(t))]\right)$ is very small, such that the influence of the quantum fluctuation of the photons cannot be ignored. Thus, the two-photon drives may effectively excite the squeezed-cavity mode, so as to induce thermal noise and two-photon correlation noise in the squeezed-cavity mode [41-44]. To minimize the influence of such noises, besides accelerating the dynamical evolution $[45,46]$, one can couple the cavity to a squeezed-vacuum reservoir [86-88] with $r_{e}=r_{\max }$ and $\varphi_{e}=\pi$ during $T / 4 \lesssim t \lesssim 3 T / 4$ [81]. Here $r_{e}$ and $\varphi_{e}$ are the squeezing parameter and the reference phase of the reservoir, respectively. In this case, the dynamics in the squeezed-light frame can be approximatively described by the standard Lindblad master equation

$$
\dot{\rho}_{S}(t) \approx i\left[\rho_{S}(t), H_{S}(t)\right]+\gamma \mathcal{D}[\sigma] \rho_{S}(t)+\kappa \mathcal{D}[a] \rho_{S}(t),
$$

where $\mathcal{D}[o] \rho_{S}(t)=o \rho_{S}(t) o^{\dagger}-\left[o^{\dagger} o \rho_{S}(t)+\rho_{S}(t) o^{\dagger} o\right] / 2$ is the standard Lindblad superoperator, $\rho_{S}(t)=$
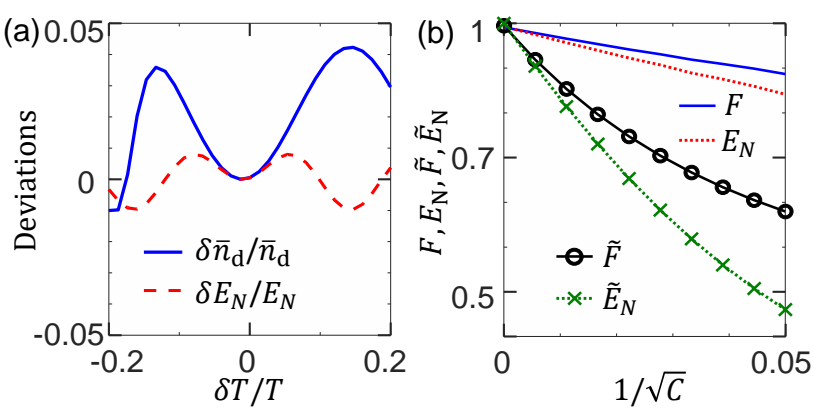

FIG. 4. (a) Deviations $\delta \bar{n}_{\mathrm{d}}$ and $\delta E_{N}$ versus $\delta T$. The STA protocol is robust against the imperfection of the total evolution time $T$. Parameters are the same as in Fig. 3(a). (b) Fidelity $F(\tilde{F})$ and logarithmic negativity $E_{N}\left(\tilde{E}_{N}\right)$ of the STA (adiabatic) protocol versus $1 / \sqrt{C}$. Here, $C=\lambda^{2} / \kappa \gamma$ is the cooperativity, and we assume the dissipation rates $\gamma=\kappa$ for simplicity. The STA and the adiabatic protocols are initially equivalent by assuming $\lambda=\tilde{\lambda}=0.045 \Delta$. The total evolution times for the entanglement generation are $T=20 / \Delta$ and $\tilde{T}=250 / \Delta$, respectively. The squeezinginduced noise is minimized by coupling the cavity to the squeezed-vacuum reservoir [81]. As a result, our STA protocol is much more robust against dissipation than the adiabatic protocol.

$S^{\dagger}(t) \rho(t) S(t)$ is the density operator in the squeezed-light frame, $\gamma$ is the spontaneous emission rate of the qubit, and $\kappa$ is the cavity decay rate.

We define the cooperativity as $C=\lambda^{2} / \kappa \gamma$ and assume $\kappa \simeq \gamma$ for simplicity. By considering the same initial parameters $\lambda=\tilde{\lambda}=0.045 \Delta$ and $r(0)=\tilde{r}(0)=0$, we compare the robustness of the STA and that of the adiabatic protocols [see Fig. 4(b)] [89]. The STA protocol is much more robust against dissipation than the adiabatic scheme, because: (i) the evolution time is significantly shortened in the STA protocol; (ii) the squeezing-induced noise can be well reduced by coupling the cavity to the squeezed-vacuum reservoir in the STA protocol. For experimentally realistic cavity QED parameters: $\Delta / 2 \pi=1 \mathrm{GHz}, \lambda / 2 \pi=45 \mathrm{MHz}$, and $\kappa / 2 \pi=\gamma / 2 \pi=2.25 \mathrm{MHz}$, the STA protocol can achieve the target state with $F \sim 90 \%$ and $E_{N} \sim 85 \%$, while the adiabatic protocol fails $\left(\tilde{F} \sim 60 \%\right.$ and $\left.\tilde{E}_{N} \sim 45 \%\right)$. Then, by measuring the qubit, we can achieve highfidelity cat states in the lab frame.

Conclusion.-We have investigated how to simulate the STA dynamics of a cavity QED system in the strong coupling regime $(\lambda>\kappa, \gamma)$ to prepare a maximally entangled cat state in the lab frame via parametric amplification. A significantly accelerated dynamics $(\sim 10$ times faster than its adiabatic counterpart) makes the system much robust against dissipation. The target state is prepared in a large-detuned JC model, which is driven by finite-duration parametric pulses. Such a setup makes our STA protocol robust against the imperfection of the evolution time. Our proposal is feasible in 
circuit QED systems, where a transmission line resonator cavity interacts with a superconducting qubit in the JC model [27, 28, 90, 91]. By attaching a superconducting quantum interference device (SQUID) to the end of the resonator [92-94], one can realize a two-photon drive (the Josephson parametric amplification process) by modulating in time the flux through the SQUID. [48, 78, 95-100]. The squeezed vacuum (reservoir) is also produced by Josephson parametric amplifiers, but with a much larger linewidth than that of the cavity [84, 98, 100-104]. This is possibly the first application of the STA protocols for the Rabi model and we hope that our protocol can find wide applications in studying light-matter interactions, specially, for the ultrastrong and deep-strong coupling regimes [9, 10].

We acknowledge helpful discussions with Yan-Xiong Du. Y.-H.C. is supported by the Japan Society for the Promotion of Science (JSPS) KAKENHI Grant No. JP19F19028. X.W. is supported by the China Postdoctoral Science Foundation No. 2018M631136, and the Natural Science Foundation of China under Grant No. 11804270. A.M. is supported by the Polish National Science Centre (NCN) under the Maestro Grant No. DEC-2019/34/A/ST2/00081. F.N. is supported in part by: NTT Research, Army Research Office (ARO) (Grant No. W911NF-18-1-0358), Japan Science and Technology Agency (JST) (via the Q-LEAP program and the CREST Grant No. JPMJCR1676), Japan Society for the Promotion of Science (JSPS) (via the KAKENHI Grant No. JP20H00134 and the JSPS-RFBR Grant No. JPJSBP120194828), the Asian Office of Aerospace Research and Development (AOARD), and the Foundational Questions Institute Fund (FQXi) via Grant No. FQXi-IAF19-06.

[1] I. I. Rabi, "On the process of space quantization," Phys. Rev. 49, 324 (1936).

[2] I. I. Rabi, "Space quantization in a gyrating magnetic field," Phys. Rev. 51, 652 (1937).

[3] D. Braak, "Integrability of the Rabi model," Phys. Rev. Lett. 107, 100401 (2011).

[4] A. Auffèves, D. Gerace, M. Richard, S. Portolan, M. F. Santos, L. C. Kwek, and C. Miniatura, Strong lightmatter coupling: from atoms to solid-state systems (World Scientific, 2013).

[5] R. H. Dicke, "Coherence in spontaneous radiation processes," Phys. Rev. 93, 99 (1954).

[6] J. J. Hopfield, "Theory of the contribution of excitons to the complex dielectric constant of crystals," Phys. Rev. 112, 1555 (1958).

[7] M. Tavis and F. W. Cummings, "Exact solution for an n-molecule-radiation-field hamiltonian," Phys. Rev. 170, 379 (1968).

[8] E. T. Jaynes and F. W. Cummings, "Comparison of quantum and semiclassical radiation theories with application to the beam maser," Proc. IEEE 51, 89
(1963).

[9] A. F. Kockum, A. Miranowicz, S. De Liberato, S. Savasta, and F. Nori, "Ultrastrong coupling between light and matter," Nat. Rev. Phys. 1, 19 (2019).

[10] P. Forn-Díaz, L. Lamata, E. Rico, J. Kono, and E. Solano, "Ultrastrong coupling regimes of light-matter interaction," Rev. Mod. Phys. 91, 025005 (2019).

[11] D. Z. Rossatto, C. J. Villas-Bôas, M. Sanz, and E. Solano, "Spectral classification of coupling regimes in the quantum Rabi model," Phys. Rev. A 96, 013849 (2017).

[12] X. Cao, J. Q. You, H. Zheng, and F. Nori, "A qubit strongly coupled to a resonant cavity: Asymmetry of the spontaneous emission spectrum beyond the rotating wave approximation," New J. Phys. 13, 073002 (2011).

[13] S. Ashhab, "Superradiance transition in a system with a single qubit and a single oscillator," Phys. Rev. A 87, 013826 (2013).

[14] S. Ashhab and F. Nori, "Qubit-oscillator systems in the ultrastrong-coupling regime and their potential for preparing nonclassical states," Phys. Rev. A 81, 042311 (2010).

[15] A. Ridolfo, M. Leib, S. Savasta, and M. J. Hartmann, "Photon blockade in the ultrastrong coupling regime," Phys. Rev. Lett. 109, 193602 (2012).

[16] A. Ridolfo, S. Savasta, and M. J. Hartmann, "Nonclassical radiation from thermal cavities in the ultrastrong coupling regime," Phys. Rev. Lett. 110, 163601 (2013).

[17] N. Shammah, N. Lambert, F. Nori, and S. De Liberato, "Superradiance with local phase-breaking effects," Phys. Rev. A 96, 023863 (2017).

[18] N. Shammah, S. Ahmed, N. Lambert, S. De Liberato, and F. Nori, "Open quantum systems with local and collective incoherent processes: Efficient numerical simulations using permutational invariance," Phys. Rev. A 98, 063815 (2018).

[19] A. P. Hines, C. M. Dawson, R. H. McKenzie, and G. J. Milburn, "Entanglement and bifurcations in Jahn-Teller models," Phys. Rev. A 70, 022303 (2004).

[20] C. Leroux, L. C. G. Govia, and A. A. Clerk, "Simple variational ground state and pure-cat-state generation in the quantum Rabi model," Phys. Rev. A 96, 043834 (2017).

[21] B. Hacker, S. Welte, S. Daiss, A. Shaukat, S. Ritter, L. Li, and G. Rempe, "Deterministic creation of entangled atom-light Schrödinger-cat states," Nat. Photon. 13, 110 (2019).

[22] L. Li, C.-L. Zou, V. V. Albert, S. Muralidharan, S. Girvin, and L. Jiang, "Cat codes with optimal decoherence suppression for a lossy Bosonic channel," Phys. Rev. Lett. 119, 030502 (2017).

[23] M. Mirrahimi, Z. Leghtas, V. V. Albert, S. Touzard, R. J. Schoelkopf, L. Jiang, and M. H. Devoret, "Dynamically protected cat-qubits: a new paradigm for universal quantum computation," New J. Phys. 16, 045014 (2014).

[24] V. V. Albert, C. Shu, S. Krastanov, C. Shen, R.-B. Liu, Z.-B. Yang, R. J. Schoelkopf, M. Mirrahimi, M. H. Devoret, and L. Jiang, "Holonomic quantum control with continuous variable systems," Phys. Rev. Lett. 116, 140502 (2016).

[25] J. Huang, Y.-H. Liu, J.-F. Huang, and J.-Q. Liao, "Generation of macroscopic entangled cat states in a 
longitudinally coupled cavity-qed model," Phys. Rev. A 101, 043841 (2020).

[26] J. Joo, W. J. Munro, and T. P. Spiller, "Quantum metrology with entangled coherent states," Phys. Rev. Lett. 107, 083601 (2011).

[27] T. Niemczyk, F. Deppe, H. Huebl, E. P. Menzel, F. Hocke, M. J. Schwarz, J. J. Garcia-Ripoll, D. Zueco, T. Hümmer, E. Solano, A. Marx, and R. Gross, "Circuit quantum electrodynamics in the ultrastrong-coupling regime," Nat. Phys. 6, 772 (2010).

[28] P. Forn-Díaz, J. Lisenfeld, D. Marcos, J. J. GarcíaRipoll, E. Solano, C. J. P. M. Harmans, and J. E. Mooij, "Observation of the Bloch-Siegert shift in a qubitoscillator system in the ultrastrong coupling regime," Phys. Rev. Lett. 105, 237001 (2010).

[29] S. J. Bosman, M. F. Gely, V. Singh, A. Bruno, D. Bothner, and G. A. Steele, "Multi-mode ultrastrong coupling in circuit quantum electrodynamics," npj Quan. Inf. 3, 46 (2017).

[30] P. Forn-Díaz, J. J. García-Ripoll, B. Peropadre, J.L. Orgiazzi, M. A. Yurtalan, R. Belyansky, C. M. Wilson, and A. Lupascu, "Ultrastrong coupling of a single artificial atom to an electromagnetic continuum in the nonperturbative regime," Nat. Phys. 13, 39 (2016).

[31] F. Yoshihara, T. Fuse, S. Ashhab, K. Kakuyanagi, S. Saito, and K. Semba, "Superconducting qubit-oscillator circuit beyond the ultrastrong-coupling regime," Nat. Phys. 13, 44 (2016).

[32] P. Forn-Díaz, G. Romero, C. J. P. M. Harmans, E. Solano, and J. E. Mooij, "Broken selection rule in the quantum Rabi model," Sci. Rep. 6, 26720 (2016).

[33] A. Baust, E. Hoffmann, M. Haeberlein, M. J. Schwarz, P. Eder, J. Goetz, F. Wulschner, E. Xie, L. Zhong, F. Quijandría, D. Zueco, J.-J. García Ripoll, L. GarcíaÁlvarez, G. Romero, E. Solano, K. G. Fedorov, E. P. Menzel, F. Deppe, A. Marx, and R. Gross, "Ultrastrong coupling in two-resonator circuit QED," Phys. Rev. B 93, 214501 (2016).

[34] Z. Chen, Y. Wang, T. Li, L. Tian, Y. Qiu, K. Inomata, F. Yoshihara, S. Han, F. Nori, J. S. Tsai, and J. Q. You, "Single-photon-driven high-order sideband transitions in an ultrastrongly coupled circuit-quantumelectrodynamics system," Phys. Rev. A 96, 012325 (2017).

[35] F. Yoshihara, T. Fuse, S. Ashhab, K. Kakuyanagi, S. Saito, and K. Semba, "Characteristic spectra of circuit quantum electrodynamics systems from the ultrastrong- to the deep-strong-coupling regime," Phys. Rev. A 95, 053824 (2017).

[36] F. Yoshihara, T. Fuse, Z. Ao, S. Ashhab, K. Kakuyanagi, S. Saito, T. Aoki, K. Koshino, and K. Semba, "Inversion of qubit energy levels in qubit-oscillator circuits in the deep-strong-coupling regime," Phys. Rev. Lett. 120, 183601 (2018).

[37] D. Ballester, G. Romero, J. J. García-Ripoll, F. Deppe, and E. Solano, "Quantum simulation of the ultrastrongcoupling dynamics in circuit quantum electrodynamics," Phys. Rev. X 2, 021007 (2012).

[38] A. Mezzacapo, U. Las Heras, J. S. Pedernales, L. DiCarlo, E. Solano, and L. Lamata, "Digital quantum Rabi and Dicke models in superconducting circuits," Sci. Rep. 4, 07482 (2014).

[39] C. Sánchez Muñoz, F. K. Anton, A. Miranowicz, and F. Nori, "Simulating ultrastrong-coupling processes breaking parity conservation in Jaynes-Cummings systems," Phys. Rev. A 102, 033716 (2020).

[40] N. K. Langford, R. Sagastizabal, M. Kounalakis, C. Dickel, A. Bruno, F. Luthi, D. J. Thoen, A. Endo, and L. DiCarlo, "Experimentally simulating the dynamics of quantum light and matter at deepstrong coupling," Nat. Commun. 8, 1715 (2017).

[41] W. Qin, A. Miranowicz, P.-B. Li, X.-Y. Lü, J. Q. You, and F. Nori, "Exponentially enhanced lightmatter interaction, cooperativities, and steady-state entanglement using parametric amplification," Phys. Rev. Lett. 120, 093601 (2018).

[42] C. Leroux, L. C. G. Govia, and A. A. Clerk, "Enhancing cavity quantum electrodynamics via antisqueezing: Synthetic ultrastrong coupling," Phys. Rev. Lett. 120, 093602 (2018).

[43] X.-Y. Lü, Y. Wu, J. R. Johansson, H. Jing, J. Zhang, and F. Nori, "Squeezed optomechanics with phasematched amplification and dissipation," Phys. Rev. Lett. 114, 093602 (2015).

[44] Y.-H. Chen, W. Qin, and F. Nori, "Fast and highfidelity generation of steady-state entanglement using pulse modulation and parametric amplification," Phys. Rev. A 100, 012339 (2019).

[45] S. C. Burd, R. Srinivas, J. J. Bollinger, A. C. Wilson, D. J. Wineland, D. Leibfried, D. H. Slichter, and D. T. C. Allcock, "Quantum amplification of mechanical oscillator motion," Science 364, 1163-1165 (2019).

[46] C. Arenz, D. I. Bondar, D. Burgarth, C. Cormick, and H. Rabitz, "Amplification of quadratic hamiltonians," Quantum 4, 271 (2020).

[47] S. C. Burd, R. Srinivas, H. M. Knaack, W. Ge, and A. C. Wilson, "Quantum amplification of bosonmediated interactions," arXiv:2009.14342 (2020).

[48] W. Qin, Y.-H. Chen, X. Wang, A. Miranowicz, and F. Nori, "Strong spin squeezing induced by weak squeezing of light inside a cavity," Nanophotonics 9, 4853-4868 (2020).

[49] B. W. Shore and P. L. Knight, "The Jaynes-Cummings model," J. Mod. Opt. 40, 1195 (1993).

[50] M. O. Scully and M. S. Zubairy, Quantum Optics (Cambridge University Press, 1997).

[51] G. S. Agarwal, Quantum Optics (Cambridge University Press, 2009).

[52] D. Guéry-Odelin, A. Ruschhaupt, A. Kiely, E. Torrontegui, S. Martínez-Garaot, and J. G. Muga, "Shortcuts to adiabaticity: Concepts, methods, and applications," Rev. Mod. Phys. 91, 045001 (2019).

[53] E. Torrontegui, S. Ibáñez, S. Martínez-Garaot, M. Modugno, A. del Campo, D. Guéry-Odelin, A. Ruschhaupt, X. Chen, and J. G. Muga, "Shortcuts to adiabaticity," Adv. At. Mol. Opt. Phys. 62, 117 (2013).

[54] X. Chen, A. Ruschhaupt, S. Schmidt, A. del Campo, D. Guéry-Odelin, and J. G. Muga, "Fast optimal frictionless atom cooling in harmonic traps: Shortcut to adiabaticity," Phys. Rev. Lett. 104, 063002 (2010).

[55] M. Demirplak and S. A. Rice, "Adiabatic population transfer with control fields," J. Phys. Chem. A 107, 9937 (2003).

[56] M. V. Berry, "Transitionless quantum driving," J. Phys. A 42, 365303 (2009).

[57] X. Chen, I. Lizuain, A. Ruschhaupt, D. Guéry-Odelin, and J. G. Muga, "Shortcut to adiabatic passage in twoand three-level atoms," Phys. Rev. Lett. 105, 123003 
(2010).

[58] A. del Campo, "Shortcuts to adiabaticity by counterdiabatic driving," Phys. Rev. Lett. 111, 100502 (2013).

[59] A. Baksic, H. Ribeiro, and A. A. Clerk, "Speeding up adiabatic quantum state transfer by using dressed states," Phys. Rev. Lett. 116, 230503 (2016).

[60] Y.-H. Chen, Y. Xia, Q.-C. Wu, B.-H. Huang, and J. Song, "Method for constructing shortcuts to adiabaticity by a substitute of counterdiabatic driving terms," Phys. Rev. A 93, 052109 (2016).

[61] Z. Zhang, T. Wang, L. Xiang, J. Yao, J. Wu, and Y. Yin, "Measuring the Berry phase in a superconducting phase qubit by a shortcut to adiabaticity," Phys. Rev. A 95, 042345 (2017).

[62] Z. Chen, Y.-H. Chen, Y. Xia, J. Song, and B.-H. Huang, "Fast generation of three-atom singlet state by transitionless quantum driving," Sci. Rep. 6, 22202 (2016).

[63] Y. Ji, J. Bian, X. Chen, J. Li, X. Nie, H. Zhou, and $\mathrm{X}$. Peng, "Experimental preparation of GreenbergerHorne-Zeilinger states in an Ising spin model by partially suppressing the nonadiabatic transitions," Phys. Rev. A 99, 032323 (2019).

[64] A. Emmanouilidou, X.-G. Zhao, P. Ao, and Q. Niu, "Steering an eigenstate to a destination," Phys. Rev. Lett. 85, 1626-1629 (2000).

[65] A. del Campo, M. M. Rams, and W. H. Zurek, "Assisted finite-rate adiabatic passage across a quantum critical point: Exact solution for the quantum Ising model," Phys. Rev. Lett. 109, 115703 (2012).

[66] O. Abah, R. Puebla, and M. Paternostro, "Quantum state engineering by shortcuts to adiabaticity in interacting spin-boson systems," Phys. Rev. Lett. 124, 180401 (2020).

[67] H. Saberi, T. Opatrný, K. Mølmer, and A. del Campo, "Adiabatic tracking of quantum many-body dynamics," Phys. Rev. A 90, 060301(R) (2014).

[68] Y.-H. Chen, Z.-C. Shi, J. Song, Y. Xia, and S.-B. Zheng, "Optimal shortcut approach based on an easily obtained intermediate Hamiltonian," Phys. Rev. A 95, 062319 (2017).

[69] T. Hatomura, "Shortcuts to adiabatic cat-state generation in bosonic Josephson junctions," New J. Phys. 20, 015010 (2018).

[70] S. Ibáñez, X. Chen, E. Torrontegui, J. G. Muga, and A. Ruschhaupt, "Multiple Schrödinger pictures and dynamics in shortcuts to adiabaticity," Phys. Rev. Lett. 109, 100403 (2012).

[71] S. Puri, S. Boutin, and A. Blais, "Engineering the quantum states of light in a Kerr-nonlinear resonator by two-photon driving," npj Quan. Inf. 3, 18 (2017).

[72] K. Funo, N. Lambert, F. Nori, and C. Flindt, "Shortcuts to adiabatic pumping in classical stochastic systems," Phys. Rev. Lett. 124, 150603 (2020).

[73] Y.-X. Du, Z.-T. Liang, Y.-C. Li, X.-X. Yue, Q.X. Lv, W. Huang, X. Chen, H. Yan, and S.-L. Zhu, "Experimental realization of stimulated Raman shortcut-to-adiabatic passage with cold atoms," Nat. Commun. 7, 12479 (2016).

[74] B. B. Zhou, A. Baksic, H. Ribeiro, C. G. Yale, F. J. Heremans, P. C. Jerger, A. Auer, G. Burkard, A. A. Clerk, and D. D. Awschalom, "Accelerated quantum control using superadiabatic dynamics in a solid-state Lambda system," Nat. Phys. 13, 330 (2016).
[75] A. Vepsäläinen, S. Danilin, and G. S. Paraoanu, "Superadiabatic population transfer in a three-level superconducting circuit," Sci. Adv. 5, eaau5999 (2019).

[76] J. Kölbl, A. Barfuss, M. S. Kasperczyk, L. Thiel, A. A. Clerk, H. Ribeiro, and P. Maletinsky, "Initialization of single spin dressed states using shortcuts to adiabaticity," Phys. Rev. Lett. 122, 090502 (2019).

[77] S. Campbell, G. De Chiara, M. Paternostro, G. M. Palma, and R. Fazio, "Shortcut to adiabaticity in the Lipkin-Meshkov-Glick model," Phys. Rev. Lett. 114, 177206 (2015).

[78] M.-A. Lemonde, N. Didier, and A. A Clerk, "Enhanced nonlinear interactions in quantum optomechanics via mechanical amplification," Nat. Commun. 7, 11338 (2016).

[79] N. N. Hegade, K. Paul, Y. Ding, M. Sanz, F. AlbarranArriagada, E. Solano, and X. Chen, "Shortcuts to adiabaticity in digitized adiabatic quantum computing," arXiv:2009.03539 (2020).

[80] M. Kolodrubetz, D. Sels, P. Mehta, and A. Polkovnikov, "Geometry and non-adiabatic response in quantum and classical systems," Phys. Rep. 697, 1 (2017).

[81] See the Supplemental Material at http://xxx which includes Ref. [50] for further details of our calculations and simulations.

[82] R. Horodecki, P. Horodecki, M. Horodecki, and K. Horodecki, "Quantum entanglement," Rev. Mod. Phys. 81, 865 (2009).

[83] J. B. Clark, F. Lecocq, R. W. Simmonds, J. Aumentado, and J. D. Teufel, "Sideband cooling beyond the quantum backaction limit with squeezed light," Nature 541, 191 (2017).

[84] K. W. Murch, S. J. Weber, K. M. Beck, E. Ginossar, and I. Siddiqi, "Reduction of the radiative decay of atomic coherence in squeezed vacuum," Nature 499, 62 (2013).

[85] H. Vahlbruch, D. Wilken, M. Mehmet, and B. Willke, "Laser power stabilization beyond the shot noise limit using squeezed light," Phys. Rev. Lett. 121, 173601 (2018).

[86] S. Ast, M. Mehmet, and R. Schnabel, "High-bandwidth squeezed light at $1550 \mathrm{~nm}$ from a compact monolithic PPKTP cavity," Optics Express 21, 13572 (2013).

[87] T. Serikawa, J.-I. Yoshikawa, K. Makino, and A. Frusawa, "Creation and measurement of broadband squeezed vacuum from a ring optical parametric oscillator," Optics Express 24, 28383 (2016).

[88] C. F. Ockeloen-Korppi, E. Damskägg, J.-M. Pirkkalainen, T. T. Heikkilä, F. Massel, and M. A. Sillanpää, "Noiseless quantum measurement and squeezing of microwave fields utilizing mechanical vibrations," Phys. Rev. Lett. 118, 103601 (2017).

[89] J. R. Johansson, P. D. Nation, and F. Nori, "QuTiP: An open-source python framework for the dynamics of open quantum systems," Compu. Phys. Commun. 183, 1760 (2012).

[90] X. Gu, A. F. Kockum, A. Miranowicz, Y.-X. Liu, and F. Nori, "Microwave photonics with superconducting quantum circuits," Phys. Rep. 718-719, 1 (2017).

[91] Z.-L. Xiang, S. Ashhab, J. Q. You, and F. Nori, "Hybrid quantum circuits: Superconducting circuits interacting with other quantum systems," Rev. Mod. Phys. 85, 623 (2013).

[92] P. D. Nation, J. R. Johansson, M. P. Blencowe, 
and F. Nori, "Colloquium: Stimulating uncertainty: Amplifying the quantum vacuum with superconducting circuits," Rev. Mod. Phys. 84, 1 (2012).

[93] M. Wallquist, V. S. Shumeiko, and G. Wendin, "Selective coupling of superconducting charge qubits mediated by a tunable stripline cavity," Phys. Rev. B 74, 224506 (2006).

[94] X. Wang, A. Miranowicz, and F. Nori, "Ideal quantum nondemolition readout of a flux qubit without Purcell limitations," Phys. Rev. Appl. 12, 064037 (2019).

[95] J. R. Johansson, G. Johansson, and F. Nori, "Optomechanical-like coupling between superconducting resonators," Phys. Rev. A 90, 053833 (2014).

[96] J. R. Johansson, G. Johansson, C. M. Wilson, and F. Nori, "Dynamical Casimir effect in a superconducting coplanar waveguide," Phys. Rev. Lett. 103, 147003 (2009).

[97] M. A. Castellanos-Beltran, K. D. Irwin, G. C. Hilton, L. R. Vale, and K. W. Lehnert, "Amplification and squeezing of quantum noise with a tunable Josephson metamaterial," Nat. Phys. 4, 929 (2008).

[98] M. A. Castellanos-Beltran and K. W. Lehnert, "Widely tunable parametric amplifier based on a superconducting quantum interference device array resonator," Appl. Phys. Lett. 91, 083509 (2007).

[99] J. R. Johansson, G. Johansson, C. M. Wilson, and F. Nori, "Dynamical Casimir effect in superconducting microwave circuits," Phys. Rev. A 82, 052509 (2010).

[100] C. Eichler and A. Wallraff, "Controlling the dynamic range of a Josephson parametric amplifier," EPJ Quantum Tech. 1, 1 (2014).

[101] M. Hatridge, R. Vijay, D. H. Slichter, J. Clarke, and I. Siddiqi, "Dispersive magnetometry with a quantum limited SQUID parametric amplifier," Phys. Rev. B 83, 134501 (2011).

[102] T. Roy, S. Kundu, M. Chand, A. M. Vadiraj, A. Ranadive, N. Nehra, M. P. Patankar, J. Aumentado, A. A. Clerk, and R. Vijay, "Broadband parametric amplification with impedance engineering: Beyond the gain-bandwidth product," Appl. Phys. Lett. 107, 262601 (2015).

[103] U. C. Mendes, S. Jezouin, P. Joyez, B. Reulet, A. Blais, F. Portier, C. Mora, and C. Altimiras, "Parametric amplification and squeezing with an ac- and dc-voltage biased superconducting junction," Phys. Rev. Appl. 11, 034035 (2019).

[104] D. M. Toyli, A. W. Eddins, S. Boutin, S. Puri, D. Hover, V. Bolkhovsky, W. D. Oliver, A. Blais, and I. Siddiqi, "Resonance fluorescence from an artificial atom in squeezed vacuum," Phys. Rev. X 6, 031004 (2016). 


\section{Supplemental Material}

In this Supplemental Material, we first discuss the influence of the nonadiabatic transition caused by mapping the system dynamics into the time-dependent squeezed-light frame. Using the transitionless algorithm, we show how to counteract such a nonadiabatic transition with an additional drive, so as to design a shortcuts-to-adiabatic passage to rapidly generate giant entangled cat states. Then, we show how to minimize the influence of the squeezing-induced fluctuation noise by coupling the cavity to a squeezed-vacuum reservoir. Thirdly, we present a possible problem in turning off the parametric drive when the target state is generated in the squeezed-light frame via the adiabatic process.

\section{S1. Effective Hamiltonian and Dissipation dynamics of the system}

\section{A. Counteracting the nonadiabatic transition caused by the time-dependent unitary transformation}

We begin with a largely detuned Jaynes-Cummings (JC) Hamiltonian driven by a time-dependent parametric (two-photon) drive $\Omega_{r}(t)$,

$$
H_{1}(t)=\Delta a^{\dagger} a-\left[\frac{\Omega_{r}(t)}{2} a^{2}-\lambda a^{\dagger} \sigma+\text { h.c. }\right] .
$$

In the time-dependent squeezed-light frame determined by the squeezing operator $S(t)=\exp \left[r(t)\left(a^{\dagger 2}-a^{2}\right) / 2\right]$, with a real squeezing parameter $r(t)$ satisfying $\tanh [2 r(t)]=\Omega_{r}(t) / \Delta$, the Hamiltonian of the system is composed of the following terms:

$$
\begin{aligned}
H_{\mathrm{S} 1}(t) & =S^{\dagger}(t) H_{1}(t) S(t)-i S^{\dagger}(t) \dot{S}(t) \\
& =H_{\mathrm{S}-\mathrm{Rabi}}(t)+H_{\mathrm{err}}(t)+H_{\mathrm{NA}}(t), \\
H_{\mathrm{S}-\mathrm{Rabi}}(t) & =\Delta \operatorname{sech}[2 r(t)] a^{\dagger} a+\lambda \exp [r(t)] \sigma_{x}\left(a^{\dagger}+a\right) / 2, \\
H_{\mathrm{err}}(t) & =-i \lambda \exp [-r(t)] \sigma_{y}\left(a^{\dagger}-a\right) / 2, \\
H_{\mathrm{NA}}(t) & =-i \dot{r}(t)\left(a^{\dagger 2}-a^{2}\right) / 2 .
\end{aligned}
$$

The Hamiltonian $H_{\mathrm{S}-\text { Rabi }}$ describes the $\sigma_{x} X$ Rabi interaction in the squeezed-light frame, where $X=\left(a+a^{\dagger}\right) / 2$ is the canonical position operator. The Hamiltonian $H_{\mathrm{err}}(t)$ describes the $\sigma_{y} Y$ interaction, where $Y=i\left(a^{\dagger}-a\right) / 2$ is the canonical momentum operator, and can be considered an error term, which can be neglected when $\lambda \ll \Delta$ and $\lambda / \Delta \ll r(t)$. When $r(t) \sim \lambda / \Delta$, the error term $H_{\text {err }}(t)$ can be neglected by applying a strong drive $\Omega \sigma_{x}(\Omega \gtrsim \Delta)$, which induces the coupling of $H_{\text {err }}(t)$ with a large detuning in the $\sigma_{y}$-direction.

The last term in $H_{\mathrm{S} 1}(t)$, i.e., $H_{\mathrm{NA}}(t)=-i S^{\dagger}(t) \dot{S}(t)$, describes a nonadiabatic transition induced by mapping the system dynamics into the time-dependent squeezed-light frame. It describes the population transfer between different basis in the squeezed-light frame. According to Berry's transitionless algorithm, we can add a term

$$
H_{\mathrm{SA}}(t)=i S^{\dagger}(t) \dot{S}(t)=i \dot{r}(t)\left(a^{\dagger 2}-a^{2}\right) / 2
$$

into the Hamiltonian $H_{\mathrm{S} 1}(t)$ to counteract the nonadiabatic transition. Then, in the laboratory frame, the additional Hamiltonian $H_{\mathrm{SA}}$ reads

$$
H_{\mathrm{add}}(t)=S(t) H_{\mathrm{SA}}(t) S^{\dagger}=i \dot{r}(t)\left(a^{\dagger 2}-a^{2}\right) / 2 .
$$

This implies that the cavity mode is subject to another two-photon drive, which has an amplitude $\Omega_{i}(t)=\dot{r}(t)$, a frequency $\omega_{p}$, and is $\pi / 2$-dephased from $\Omega_{r}(t)$. By adding this additional Hamiltonian $H_{\text {add }}(t)$ into the Hamiltonian $H_{1}(t)$, we obtain the Hamiltonian $H_{0}(t)$ required for the STA protocol, i.e., the Hamiltonian of Eq. (6) of the main text:

$$
H_{0}(t)=\Delta a^{\dagger} a+\Omega \sigma_{x}-\left[\frac{\Omega_{r}(t)+i \Omega_{i}(t)}{2} a^{2}-\lambda a^{\dagger} \sigma+\text { h.c. }\right] .
$$

Then, we are allowed to rapidly change the squeezing parameter $r(t)$, such that we can quickly adjust the effective qubit-cavity coupling $\lambda \exp [r(t)] / 2$ in the squeezed-light frame.

This is very important, because applying the STA protocol requires to rapidly change the control parameter, i.e., the normalized coupling strength. 


\section{B. STA process with parametric drivings}

To construct the STA passage, we divide the Hamiltonian $H_{S}(t)$ into two parts:

$$
H_{S}(t)=H_{\text {ref }}(t)+H_{\text {aux }}(t)
$$

Here, the Hamiltonian

$$
H_{\mathrm{ref}}(t)=\Delta \operatorname{sech}[2 r(t)] a^{\dagger} a+\sigma_{x}\left[\chi(t) a^{\dagger}+\chi^{*}(t) a\right],
$$

is considered as the reference Hamiltonian [with an undetermined parameter $\chi(t)$ ] for constructing shortcuts,

$$
H_{\mathrm{aux}}(t)=\frac{\lambda e^{r(t)}}{2} \sigma_{x}\left(a^{\dagger}+a\right)-\sigma_{x}\left[\chi(t) a^{\dagger}+\chi^{*}(t) a\right]
$$

is an auxiliary Hamiltonian. The reference Hamiltonian $H_{\text {ref }}(t)$ takes the same form as the Rabi Hamiltonian $H_{R}(t)$ [Eq. (1) of the main text], i.e., $H_{\text {ref }}(t): \Rightarrow H_{R}(t)$, by setting:

$$
\omega_{q} \ll \omega_{c}, \quad \omega_{c}: \Rightarrow \Delta \operatorname{sech}[2 r(t)], \quad g: \Rightarrow \chi(t) .
$$

Then, when we choose the parameters to satisfy

$$
\eta(t)=\frac{g}{\omega_{c}}=\frac{\chi(t)}{\Delta \operatorname{sech}[2 r(t)]}, \quad \dot{\eta}(t)=\frac{i}{2}\left[\lambda e^{r(t)}-2 \chi(t)\right],
$$

$H_{\text {aux }}(t)$ is exactly the CD driving Hamiltonian for the reference Hamiltonian $H_{\text {ref }}(t)$, i.e., $H_{\text {aux }}(t): \Rightarrow H_{\mathrm{CD}}(t)$. Hence, according to the transitionless algorithm, the CD driving Hamiltonian $H_{\text {aux }}(t)$ can actually drive the system to evolve along an eigenstate of $H_{\text {ref }}(t)$. The evolution path for our STA protocol is then given as (in the squeezed-light frame)

$$
\left|E_{0}(t)\right\rangle_{S}=\frac{1}{\sqrt{2}}\left[\left|{ }_{x}\right\rangle|-\eta(t)\rangle+\left|{ }_{x}\right\rangle|\eta(t)\rangle\right],
$$

where $\left| \pm_{x}\right\rangle$ are the eigenstates of the Pauli matrix $\sigma_{x}$. In the lab frame, the STA evolution path is $S[r(t)]\left|E_{0}(t)\right\rangle_{S}$. After some algebra, we can counteract the undetermined parameter $\chi(t)$ and obtain the equations of motion for the coherent state amplitude $\eta(t)$ :

$$
\begin{aligned}
\operatorname{Re}[\dot{\eta}(t)] & =\Delta \operatorname{Im}[\eta(t)] \operatorname{sech} 2 r(t), \\
\operatorname{Im}[\dot{\eta}(t)] & =\frac{\lambda}{2} \exp [r(t)]-\Delta \operatorname{Re}[\eta(t)] \operatorname{sech} 2 r(t) .
\end{aligned}
$$

Thus, Eq. (9) of the main text is obtained. The final state in the laboratory frame is

$$
S\left(t_{f}\right)\left|E_{0}\left(t_{f}\right)\right\rangle_{S}=\frac{1}{\sqrt{2}}\left(\left|+_{x}\right\rangle\left|-\eta\left(t_{f}\right)\right\rangle+\left|-_{x}\right\rangle\left|\eta\left(t_{f}\right)\right\rangle\right),
$$

which is an entangled cat state. Here, $S\left(t_{f}\right)=1$ is given according to $r\left(t_{f}\right)=0$.

\section{Minimizing the influence of the squeezing-induced fluctuation noise}

The Markovian master equation, for a cavity interacting with a broadband squeezed-vacuum reservoir (at zero temperature with squeezing parameter $r_{e}$ and reference phase $\varphi_{e}$ ), has been well studied (see, e.g., Ref. [50]). For our STA protocol, when the cavity couples to the squeezed-vacuum reservoir, the master equation in the laboratory frame is

$$
\begin{aligned}
\dot{\rho}(t)= & i\left[\rho(t), H_{0}(t)\right]+\frac{1}{2}\left[2 L_{\gamma} \rho(t) L_{\gamma}^{\dagger}-L_{\gamma}^{\dagger} L_{\gamma} \rho(t)-\rho(t) L_{\gamma}^{\dagger} L_{\gamma}\right] \\
& +\frac{1}{2}(N+1)\left[2 L_{\kappa} \rho(t) L_{\kappa}^{\dagger}-L_{\kappa}^{\dagger} L_{\kappa} \rho(t)-\rho(t) L_{\kappa}^{\dagger} L_{\kappa}\right] \\
& +\frac{1}{2} N\left[2 L_{\kappa}^{\dagger} \rho(t) L_{\kappa}-L_{\kappa} L_{\kappa}^{\dagger} \rho(t)-\rho(t) L_{\kappa} L_{\kappa}^{\dagger}\right] \\
& -\frac{1}{2} M\left[2 L_{\kappa}^{\dagger} \rho(t) L_{\kappa}^{\dagger}-L_{\kappa}^{\dagger} L_{\kappa}^{\dagger} \rho(t)-\rho(t) L_{\kappa}^{\dagger} L_{\kappa}^{\dagger}\right] \\
& -\frac{1}{2} M^{*}\left[2 L_{\kappa} \rho(t) L_{\kappa}-L_{\kappa} L_{\kappa} \rho(t)-\rho(t) L_{\kappa} L_{\kappa}\right] .
\end{aligned}
$$



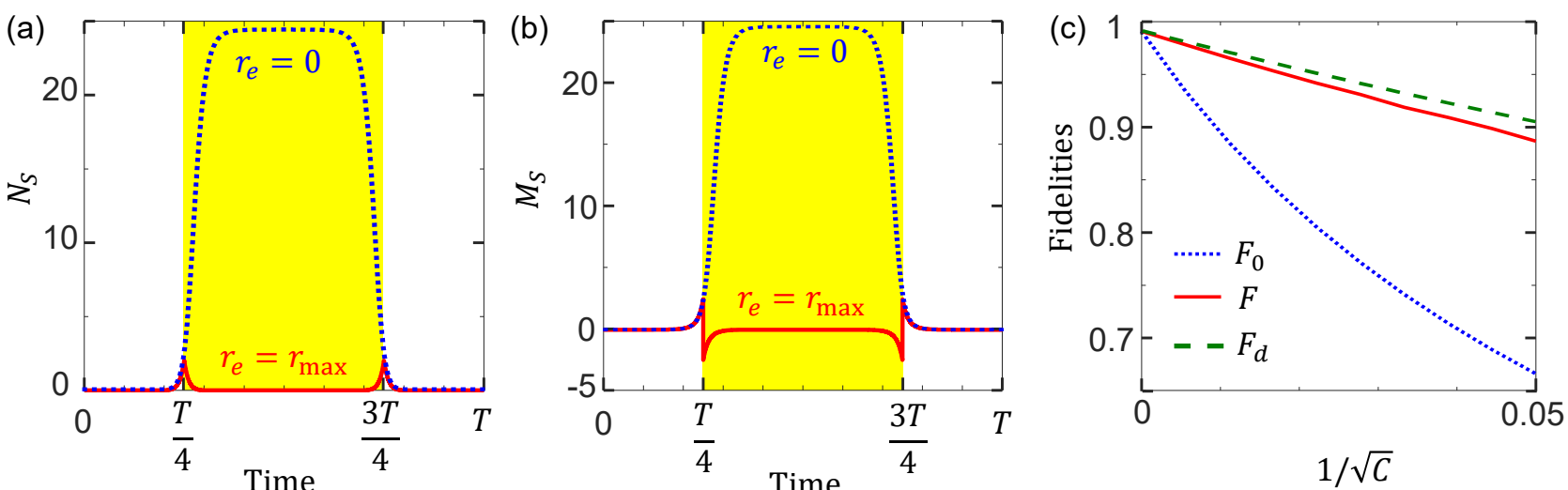

FIG. S1. Parameters (a) $N_{S}$ and (b) $M_{S}$ characterizing the squeezing-induced noise for $r_{\max }=2.3$ and $\Omega=0$. Blue-dotted curve in (a) [(b)]: Parameter $N_{S}\left(M_{S}\right)$ without coupling the cavity to the squeezed-vacuum reservoir (i.e., $\left.r_{e}=0\right)$; Red-solid curve in (a) [(b)]: Parameter $N_{S}\left(M_{S}\right)$ when the system is coupled to the squeezed-vacuum reservoir during $T / 4 \lesssim t \lesssim 3 T / 4$ [i.e., $r_{e}$ is given according to Eq. (S21)]. The yellow-shaded area in (a) or (b) denotes when the cavity is coupled to the squeezed-vacuum reservoir. (c) Fidelities of the ground state $|G\rangle$ versus $1 / \sqrt{C}$ calculated by: (blue-dotted curve representing $\left.F_{0}\right)$ the noise-included master equation in Eq. (S16) when $r_{e}=0$; (red-solid curve representing $F$ ) the noise-included master equation when coupling the cavity to the squeezed-vacuum; (green-dashed curve representing $F_{d}$ ) the effective master equation in Eq. (S23). The parameter $C=\lambda^{2} / \kappa \gamma$ is the cooperativity, and we assume the dissipation rates $\gamma=\kappa$ for simplicity.

Here, $L_{\gamma}=\sqrt{\gamma} \sigma$ and $L_{\kappa}=\sqrt{\kappa} a$ describe the qubit and cavity decays, with decay rates $\gamma$ and $\kappa$, respectively. The parameters

$$
N=\sinh ^{2}\left(r_{e}\right), \quad \text { and } \quad M=\cosh \left(r_{e}\right) \sinh \left(r_{e}\right) \exp \left(-i \varphi_{e}\right),
$$

describe thermal noise and two-photon correlation noise caused by the squeezed-vacuum reservoir, respectively.

By mapping the system dynamics into the time-dependent squeezed-light frame with $S(t)$, the master equation becomes

$$
\begin{aligned}
\dot{\rho}_{S}(t)= & \left.i \rho_{S}(t), H_{\mathrm{S}-\operatorname{Rabi}}(t)+H_{\mathrm{err}}(t)\right]+\frac{1}{2}\left[2 L_{\gamma} \rho_{S}(t) L_{\gamma}^{\dagger}-L_{\gamma}^{\dagger} L_{\gamma} \rho_{S}(t)-\rho_{S}(t) L_{\gamma}^{\dagger} L_{\gamma}\right] \\
& +\frac{1}{2}\left(N_{S}+1\right)\left[2 L_{\kappa} \rho_{S}(t) L_{\kappa}^{\dagger}-L_{\kappa}^{\dagger} L_{\kappa} \rho_{S}(t)-\rho_{S}(t) L_{\kappa}^{\dagger} L_{\kappa}\right] \\
& +\frac{1}{2} N_{S}\left[2 L_{\kappa}^{\dagger} \rho_{S}(t) L_{\kappa}-L_{\kappa} L_{\kappa}^{\dagger} \rho_{S}(t)-\rho_{S}(t) L_{\kappa} L_{\kappa}^{\dagger}\right] \\
& -\frac{1}{2} M_{S}\left[2 L_{\kappa}^{\dagger} \rho_{S}(t) L_{\kappa}^{\dagger}-L_{\kappa}^{\dagger} L_{\kappa}^{\dagger} \rho_{S}(t)-\rho_{S}(t) L_{\kappa}^{\dagger} L_{\kappa}^{\dagger}\right] \\
& -\frac{1}{2} M_{S}^{*}\left[2 L_{\kappa} \rho_{S}(t) L_{\kappa}-L_{\kappa} L_{\kappa} \rho_{S}(t)-\rho_{S}(t) L_{\kappa} L_{\kappa}\right]
\end{aligned}
$$

where $\rho_{S}(t)=S^{\dagger}(t) \rho(t) S(t)$ is the density operator of the system in the squeezed-light frame, and

$$
\begin{aligned}
N_{S}= & \cosh ^{2}[r(t)] \sinh ^{2}\left(r_{e}\right)+\sinh ^{2}[r(t)] \cosh ^{2}\left(r_{e}\right)+\frac{1}{2} \sinh [2 r(t)] \sinh \left(2 r_{e}\right) \cos \left(\varphi_{e}\right), \\
M_{S}= & \left\{\sinh [r(t)] \cosh \left(r_{e}\right)+\exp \left(-i \varphi_{e}\right) \cosh [r(t)] \sinh \left(r_{e}\right)\right\} \\
& \times\left\{\cosh [r(t)] \cosh \left(r_{e}\right)+\exp \left(i \varphi_{e}\right) \sinh [r(t)] \sinh \left(r_{e}\right)\right\},
\end{aligned}
$$

characterize additional noises of the system in the squeezed-light frame. When $r_{e}=0, N_{S}$ and $M_{S}$ characterize the squeezing-induced noise. For simplicity, we can assume $\varphi_{e}=\pi$, and obtain

$$
N_{S}=\sinh ^{2}\left[r_{S}(t)\right], \quad \text { and } \quad M_{S}=\cosh \left[r_{S}(t)\right] \sinh \left[r_{S}(t)\right],
$$

where $r_{S}(t)=r(t)-r_{e}$. Then, to minimize the parameters $\left|N_{S}\right|$ and $\left|M_{S}\right|$, we need to minimize the parameter $\left|r_{S}(t)\right|$.

The waveform of $r(t)$ of the STA protocol is approximately a square wave when

$$
r(t)=\frac{r_{\max }}{1+\exp \left[f_{0} \cos (2 \pi t / T)\right]},
$$



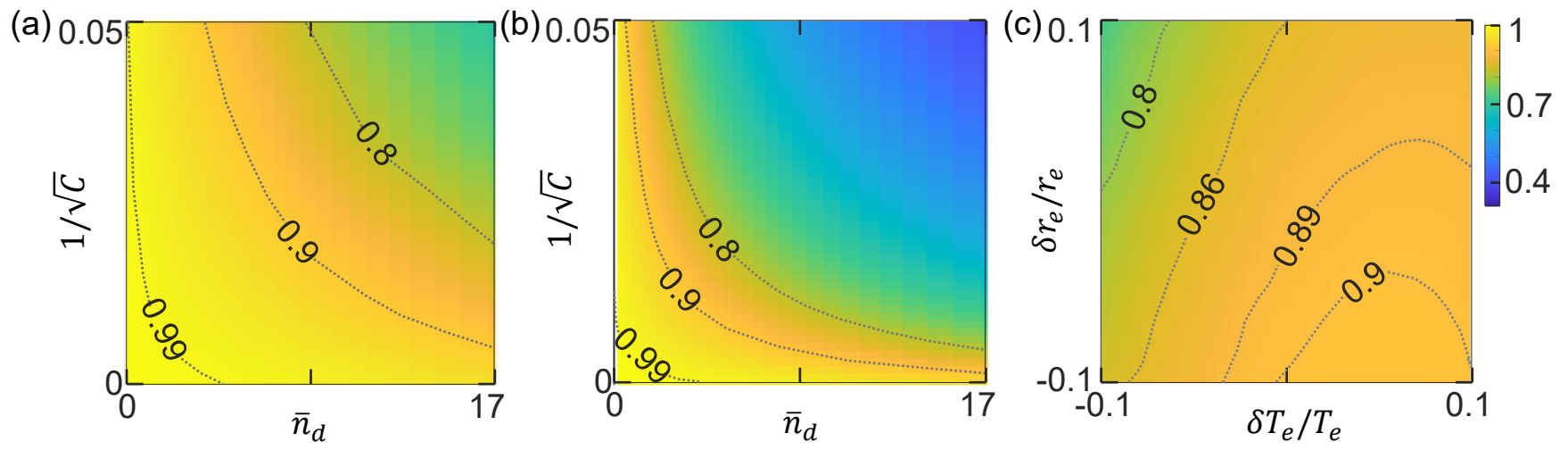

FIG. S2. blue Fidelities $F$ of the entangled cat state for the STA protocol with $T=20 / \Delta$ and $\lambda=0.045 \Delta$ : (a) [(b)] Fidelity $F$ versus the cooperativity $C$ and the predicted mean photon number $\bar{n}_{d}=|\eta|^{2}$, when the cavity is coupled (decoupled) to the squeezed-vacuum reservoir. For the panels (a) and (b), we choose the squeezing parameter $r_{\max } \in[0,3]$ corresponding to the $\bar{n}_{d}=|\eta|^{2} \in[0,17]$. (c) Fidelity $F$ versus imperfections of the parameters $r_{e}$ and $T_{e}$. For the panel (c), the squeezing parameter is $r_{\max }=2.3$.

where $f_{0}=10$ controls the initial and final values of the squeezing parameter $r(t)$. Substituting Eq. (S19) into Eq. (S18) and assuming $r_{e}=0$, in Figs. S1(a) and S1(b), we show the parameters $N_{S}$ and $M_{S}$ describing the squeezing-induced noise (see the blue-dotted curves). As shown, the squeezing-induced noise affects the system dynamics especially when $r(t)$ reaches its maximum value $r_{\max }$, i.e., $r(t) \approx r_{\max }$. We accordingly calculate the fidelity $F_{0}=\left|\left\langle G\left|\rho_{S}\left(t_{f}\right)\right| G\right\rangle\right|$ to show the influence of the squeezing-induced noise [see the blue-dotted curve in Fig. S1(c)]. Here, $|G\rangle$ is the ground state of the Rabi model in the DSC regime [see Eq. (2) of the main text]. The fidelity $F_{0}$ decreases very fast when the dissipation increases.

To minimize the parameter $\left|r_{S}(t)\right|$, according to the properties of $\cos (2 \pi t / T)$, we can choose

$$
r_{e}=\left\{\begin{aligned}
0, & (0 \lesssim t \lesssim T / 4) \\
r_{\max }, & (T / 4 \lesssim t \lesssim 3 T / 4) \\
0, & (3 T / 4 \lesssim t \lesssim T)
\end{aligned}\right.
$$

i.e., the total interaction time between the cavity and the squeezed-vacuum reservoir is $T_{e}=T / 2$, resulting in

$$
r_{S}(t)=\left\{\begin{array}{cl}
\frac{r_{\max }}{1+\exp \left[f_{0} \cos (2 \pi t / T)\right]}, & (0 \lesssim t \lesssim T / 4) \\
\frac{-r_{\max }}{1+\exp \left[-f_{0} \cos (2 \pi t / T)\right]}, & (T / 4 \lesssim t \lesssim 3 T / 4) \\
\frac{r_{\max }}{1+\exp \left[f_{0} \cos (2 \pi t / T)\right]}, & (3 T / 4 \lesssim t \lesssim T)
\end{array}\right.
$$

Then, substituting Eq. (S21) into Eq. (S18), we plot the parameters $N_{S}$ and $M_{S}$ [see the red-solid curves in Fig. S1(a) and $\mathrm{S} 1(\mathrm{~b})]$. We can accordingly calculate the average values

$$
A_{N_{S}}=\frac{1}{T} \int_{0}^{t_{f}}\left|N_{S}\right| d t \approx 0.08, \quad \text { and } \quad A_{M_{S}}=\frac{1}{T} \int_{0}^{t_{f}}\left|M_{S}\right| d t \approx 0.14,
$$

which means that the additional noises in Eq. (S16) weakly affect the system dynamics. Thus, the fidelity of the target state $|G\rangle$ is significantly improved [see the red-solid curve in Fig. S1(c)], e.g., from $\sim 65 \%$ to $\sim 89 \%$ when $1 / \sqrt{C}=0.05$. When the desired mean photon number $\bar{n}_{d}$ of the target state increases, the influence of the cavity loss increases [see Figs. S3(a) and S3(b)]. These figures show the fidelities of the target state when the cavity is coupled and decoupled to the squeezed-vacuum reservoir, respectively. According to the comparison between Figs. S1(a) and S1(b), coupling the cavity to the squeezed-vacuum reservoir can effectively suppress the influence of the cavity loss. Thus, the giant $\left(\bar{n}_{d} \gtrsim 10\right)$ entangled cat states can be generated with a high fidelity. By defining the imperfection of a 

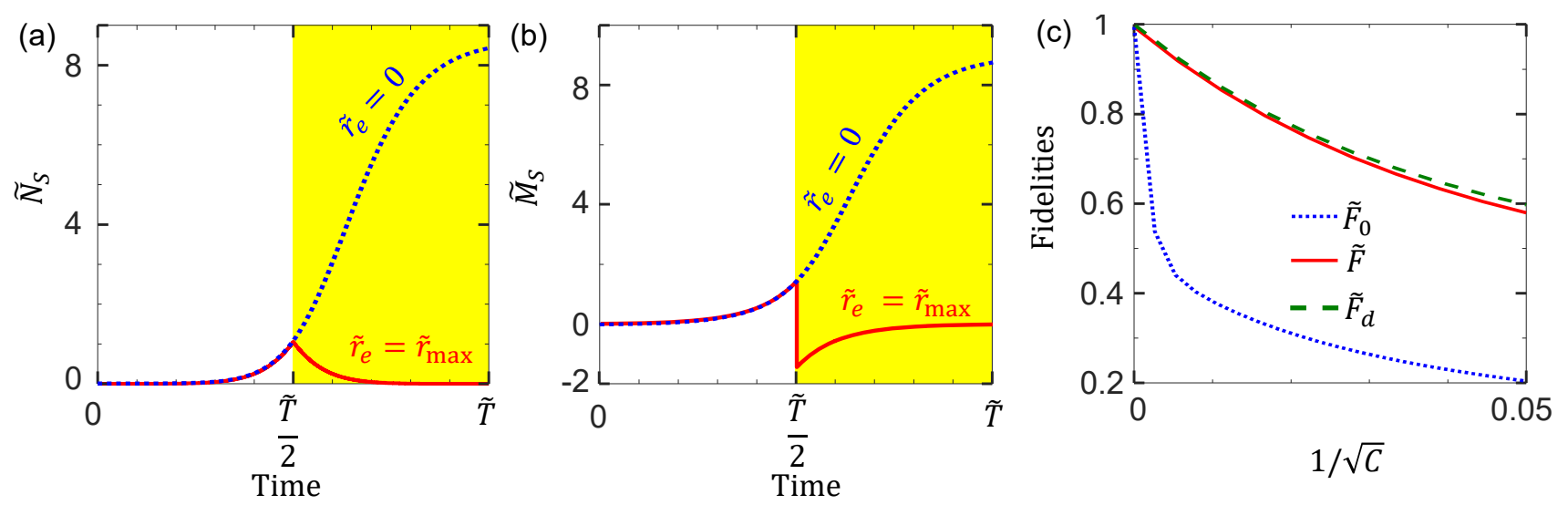

FIG. S3. Parameters (a) $\tilde{N}_{S}$ and (b) $\tilde{M}_{S}$ characterizing the squeezing-induced noise for $\tilde{r}_{\max }=1.8$. Blue-dotted curve in (a) [(b)]: parameter $\tilde{N}_{S}\left(\tilde{M}_{S}\right)$ without coupling the cavity to the squeezed-vacuum reservoir (i.e., $\tilde{r}_{e}=0$ ); Red-solid curve in (a) [(b)]: parameter $\tilde{N}_{S}\left(\tilde{M}_{S}\right)$ when the system is coupled to the squeezed-vacuum reservoir during $\tilde{T} / 2 \lesssim t \lesssim \tilde{T}$ [i.e., $\tilde{r}_{e}$ is given according to Eq. (S25)]. The yellow-shaded area in (a) or (b) denotes that the cavity is coupled to the squeezed-vacuum reservoir. (c) Fidelities of the squeezed ground state $|S G\rangle=S\left(t_{f}\right)|G\rangle$ versus $1 / \sqrt{C}$ calculated by: (blue-dotted curve representing $\tilde{F}_{0}$ ) the noise-included master equation in Eq. (S16) when $r_{e}=0$; (red-solid curve representing $\tilde{F}$ ) the noise-included master equation when coupling the cavity to the squeezed-vacuum reservoir; (green-dashed curve representing $\tilde{F}_{d}$ ) the effective master equation in Eq. (S23).

parameter $*$ as $\delta *=*^{\prime}-*$, the influence of the imperfections of the parameters $T_{e}$ and $r_{e}$ is shown in Fig. S1(c). This figure shows that, slightly decreasing the squeezing parameter $r_{e}$ or increasing the interaction time $T_{e}$ can improve the fidelity $F$. Note that a $10 \%$ imperfection of the parameter $r_{e}$ only causes a $3 \%$ change in the fidelity, thus the STA protocol is mostly insensitive to the imperfections of the parameter $r_{e}$. When the interaction time $T_{e}$ between the cavity and the squeezed-vacuum reservoir is long enough, our STA protocol is mostly insensitive to the imperfections of the parameter $T_{e}$.

When coupling the cavity to the squeezed-vacuum reservoir during $T / 4 \lesssim t \lesssim 3 T / 4$, the evolution can be approximately described by the standard Lindblad master equation

$$
\dot{\rho}_{S}(t) \approx i\left[\rho_{S}(t), H_{\mathrm{S}-\mathrm{Rabi}}(t)\right]+\frac{1}{2} \sum_{m=\kappa, \gamma}\left[2 L_{m} \rho_{S}(t) L_{m}^{\dagger}-L_{m}^{\dagger} L_{m} \rho_{S}(t)-\rho_{S}(t) L_{m}^{\dagger} L_{m}\right]
$$

which is Eq. (10) of the main text. As shown in Fig. S1(c), the Lindblad master equation can well describe the dynamics when the cavity is coupled to the squeezed-vacuum reservoir.

This strategy is also applicable in the adiabatic protocol to minimize the influence of the squeezing-induced noise. For the adiabatic protocol, the squeezing parameter $\tilde{r}(t)$ is

$$
\tilde{r}(t)=\frac{\tilde{r}_{\max }}{1+\exp \left[\tilde{f}_{0}(1 / 2-t / \tilde{T})\right]},
$$

where $\tilde{f}_{0}=10$ controls the initial and final values of $\tilde{r}(t)$. Substituting Eq. (S24) into Eq. (S17) and assuming $\tilde{r}_{e}=0$, we plot the parameters $\tilde{N}_{S}$ and $\tilde{M}_{S}$ in Figs. S3(a) and S3(b). We denote $\tilde{*}(*=r, T, \ldots)$ to represent the parameters used in the adiabatic protocol. The parameter $\tilde{*}$ has the same physical meaning as $*$. Due to the squeezing-induced noise, the adiabatic protocol becomes unreliable for the finite cooperativity $C$ [see the blue-dotted curve in Fig. S3(c)].

To minimize the parameters $\left|\tilde{N}_{S}\right|$ and $\left|\tilde{M}_{S}\right|$, we can assume

$$
\tilde{r}_{e}=\left\{\begin{array}{rr}
0, & (0 \lesssim t \lesssim \tilde{T} / 2) \\
\tilde{r}_{\max }, & (\tilde{T} / 2 \lesssim t \lesssim \tilde{T})
\end{array}\right.
$$



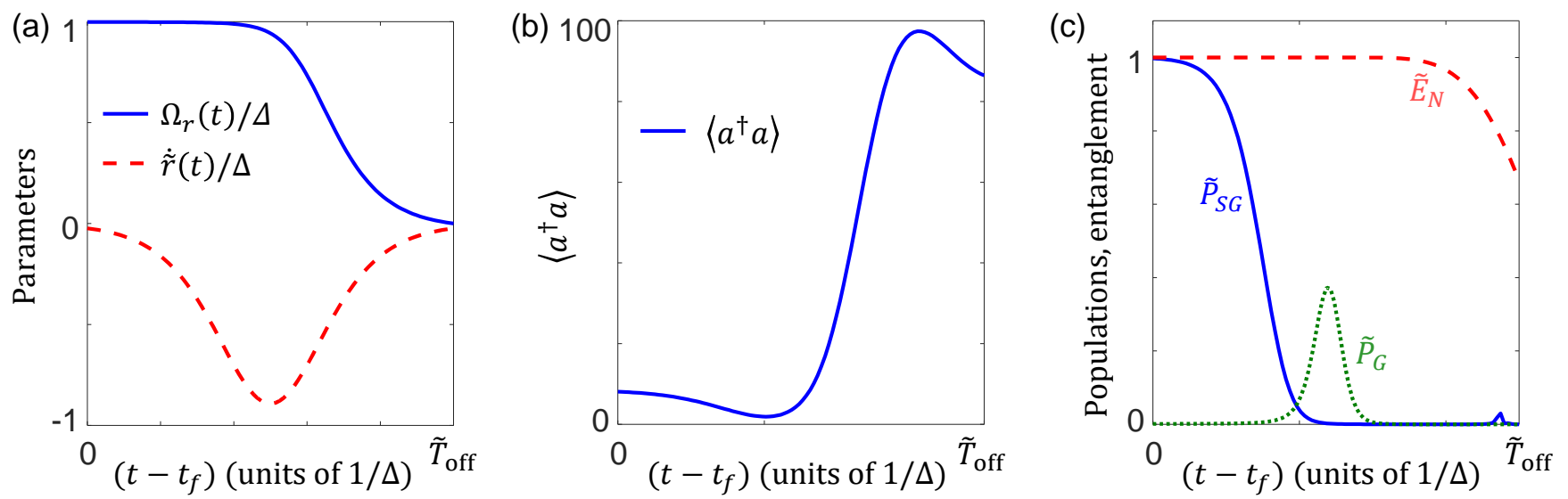

FIG. S4. System evolution during turning off the parametric drive in the adiabatic protocol for $t>t_{f}$. (a) Amplitude of the parametric drive $\Omega_{r}(t)$ and the changing rate $\dot{\tilde{r}}(t)$ of the squeezing parameter $r(t)$. (b) Mean photon number $\left\langle a^{\dagger} a\right\rangle$ of the system. (c) Blue-solid curve: the population $\tilde{P}_{S G}$ of the squeezed state $|S G\rangle$; Green-dotted curve: the population $\tilde{P}_{G}$ of the ground state $|G\rangle$; Red-dashed curve: the entanglement cost (characterized by the logarithmic negativity $\tilde{E}_{N}$ ). The time required to turn off the parametric drive is assumed to be $\tilde{T}_{\text {off }}=5 / \Delta$.

resulting in

$$
\tilde{r}_{S}(t)=\left\{\begin{array}{cl}
\frac{\tilde{r}_{\max }}{1+\exp \left[\tilde{f}_{0}(1 / 2-t / \tilde{T})\right]}, & (0 \lesssim t \lesssim \tilde{T} / 2) \\
\frac{-\tilde{r}_{\max }}{1+\exp \left[-\tilde{f}_{0}(1 / 2-t / \tilde{T})\right]}, & (\tilde{T} / 2 \lesssim t \lesssim \tilde{T})
\end{array}\right.
$$

Accordingly, the average values of $\left|\tilde{N}_{S}\right|$ and $\left|\tilde{M}_{S}\right|$ are

$$
\tilde{A}_{N_{S}}=\frac{1}{\tilde{T}} \int_{0}^{t_{f}}\left|\tilde{N}_{S}\right| d t \approx 0.14, \quad \text { and } \quad \tilde{A}_{M_{S}}=\frac{1}{\tilde{T}} \int_{0}^{t_{f}}\left|\tilde{M}_{S}\right| d t \approx 0.3
$$

respectively. Thus, the additional noises characterized by $\tilde{N}_{S}$ and $\tilde{M}_{S}$ can be suppressed as shown in Fig. S3(a) and S3(b). The fidelity of the squeezed ground state $|S G\rangle=S\left(t_{f}\right)|G\rangle$ is improved [see the red-solid curve in Fig. S3(c)]. However, due to

$$
\tilde{A}_{N_{S}}>A_{N_{S}}, \quad \tilde{A}_{M_{S}}>A_{M_{S}}, \quad \text { and } \tilde{T} \gg T,
$$

the squeezing-induced noise still affects the adiabatic protocol more seriously than the STA protocol. Thus, the fidelity of the adiabatic protocol is much lower than the STA method, according to the comparison between Figs. S1(c) and S3(c).

\section{S2. A Possible Problem caused by turning off the parametric drive in the adiabatic protocol}

The nonadiabatic transition $H_{\mathrm{NA}}(t)$ also causes the main problem of how to turn off the parametric drive. In the adiabatic protocol discussed in the main text, the amplitude of the parametric drive $\Omega_{r}(t)$ reaches the peak value at the time $t_{f}$, i.e., $\Omega_{r}\left(t_{f}\right)=\Omega_{\max }$. Meanwhile, the maximally entangled cat state is prepared in the squeezed frame. In the laboratory frame, the final state corresponds to the qubit being entangled with the squeezed and displaced cavity pointer states, i.e., $|S G\rangle$. To smoothly and rapidly turn off the parametric drive, we can assume

$$
\tilde{r}(t)=\frac{1}{2} \frac{\operatorname{arctanh}\left(\Omega_{\max } / \Delta\right)}{1+\exp \left\{10\left[-\left(t-t_{f}\right) / \tilde{T}_{\text {off }}+1 / 3\right]\right\}}, \quad\left(t \geq t_{f}\right)
$$

corresponding to

$$
\tilde{r}\left(t_{f}\right)=\frac{1}{2} \operatorname{arctanh}\left(\Omega_{\text {max }} / \Delta\right), \quad \tilde{r}\left(t_{f}+\tilde{T}_{\text {off }}\right) \simeq 0, \quad \dot{\tilde{r}}\left(t_{f}\right) \simeq 0, \quad \dot{\tilde{r}}\left(t_{f}+\tilde{T}_{\text {off }}\right) \simeq 0 .
$$


Here, $\tilde{T}_{\text {off }}$ is the operation time required to turn off the parametric drive.

Assuming $\tilde{T}_{\text {off }}=5 / \Delta$ as an example, we show $\Omega_{r}(t)$ and $\dot{\tilde{r}}(t)$ versus time in Fig. S4(a). Due to $\dot{\tilde{r}}(t) \neq 0$, the nonadiabatic transition $H_{\mathrm{NA}}(t)$ can pump many photons into the cavity. By substituting Eq. (S29) into Eq. (S2), and assuming the system is in the squeezed ground state $|S G\rangle$ at the time $t_{f}$, we show the instantaneous mean photon number $\left\langle a^{\dagger} a\right\rangle$ when $t>t_{f}$ in Fig. S4(b). We find that $\left\langle a^{\dagger} a\right\rangle$ increases sharply when $\Omega_{r}(t)$ decreases. When the parametric drive is turned off, i.e., $\Omega_{r}(t)=0$, the desired entangled state does not exist any longer [see in Fig. S4(c)]. Both populations of the squeezed ground state $|S G\rangle\left(\tilde{P}_{S G}\right)$ and the state $|G\rangle\left(\tilde{P}_{G}\right)$ reach 0 when the parametric drive is turned off [see the blue-solid and green-dotted curves in Fig. S4(c)]. The entanglement cost (characterized by the logarithmic negativity $\tilde{E}_{N}$ ) decreases to a low value, i.e., $\tilde{E}_{N} \sim 70 \%$. That is, the state of the system after turning off the parametric drive is unpredictable. 